\title{
Über Versuche zur Darstellung von Derivaten des Diamido-acetylens.
}

(4. Mitteilung über Acetylenderivate ${ }^{1}$ )

von

Paul Ruggli.

(27. v. 20.)

Im Folgenden sollen einige Versuche beschrieben werdẹn, welche den $\mathrm{Zweck}$ verfolgen, zu Derivaten des unbekannten Diamido-acetylens $\mathrm{H}_{2} \mathrm{~N}-\mathrm{C} \equiv \mathrm{C}-\mathrm{NH}_{2}$ zu gelangen, in welchen die Amidowasserstoffatome teilweise oder ganz durch andere Gruppen ersetzt sind. Aus der Literatur ist mir nur eine Verbindung bekannt, welche überhaupt ein Stickstoffatom direkt an der Acetylenbindung trägt, das Trimethyl-äthinyl-ammoniumhydroxyd von Bode ${ }^{2}$.<smiles>C#C[V](C)(C)O</smiles>

Formal erschien es naheliegend, das leicht zugängliche Acetylen-tetrachlorid als Ausgangsmaterial zu verwenden, indem man jederseits ein Chloratom mit einem sekundären Amin unter Chlorwasserstoffabspaltung umsetzt und nachträglich zwei weitere Molekeln Chlorwasserstoff abspaltet:

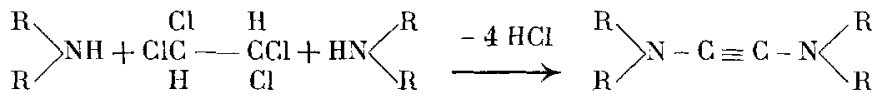

Basische Amine sind hierbei ausgeschlossen, da sie nur Chlorwasserstoff abspalten unter Bildung des weniger reaktionsfähigen Trichlor-äthylens $\left.{ }^{3}\right) \mathrm{HClC}=\mathrm{CGl}_{2}$. Es wurden daher Versuche mit dem nicht basischen Diphenylamin angestellt, doch erfolgte auch hier nur Abspaltung von Chlorwasserstoff ohne Kondensation.

1) Vergl. A. 392, 92 (1912); 399, 174 (1913); 412, 1 (1916).

2) A. 267, 286 (1892); die Substanz wurde nur in Form von Doppelsalzen analysiert; sie ist ein Analogon des Neurins und giftiger als dieses.

3) Dagegen reagiert das Trichloräthylen bekanntlich glatt mit Natriumalkoholat zu Dichlor-vinyl-äthyläther. 
Mehr Aussicht bot eine andere Möglichkeit: der beiderseitige $A b b a u$ der Acetylen-dicarbonsäure zum nächst niedrigeren Amin, wobei formal Diamido-acetylen entstände. Für einen solchen Abbau haben wir drei Methoden zur Verfügung: den Abbau der Säureamide mit Brom und Alkali nach Hofmann, den Weg über die Säurehydrazide und Azide und Verkochen derselben mit Alkohol nach Curtius, und schliesslich die Spaltung der benzoylierten Hydroxamsäuren nach Lossen ${ }^{1}$ ).

Die Acetylen-dicarbonsäure selber resp. ihr Ester eignet sich wenig für diese Versuche, da die bei den genannten Reaktionen erforderlichen Reagenzien sich meist an die Acetylenbindung anlagern. So gibt bekanntlich Acetylen-dicarbonsäureester mit Hydrazin Pyrazolon-carbonsäureester \%). Hydroxylamin gibt, wie ich feststellte, mit Acetylen-dicarbonsäureester allerdings eine Hydroxamsäure, doch ist dieselbe nur in Form ihrer Salze beständig und zersetzt sich beim Versuch, sie zu isolieren. Der Hofmann'sche Abbau endlich ist bei ungesättigten Säuren nicht empfehlenswert ${ }^{3}$ ).

Da $A . v$. Baeyer $^{4}$ ) seinerzeit beobachtete, dass Acetylendicarbonsäure (und ebenso die Diacetylen-dicarbonsäure) mit alkoholischer Salzsäure bei $0^{\circ}$ ohne Addition verestert werden kann, dass sie also Chlorwasserstoff unter diesen Umständen nicht addiert, so wurde versucht, ob sich durch vorsichtige Behandlung mit Phosphorpentachlorid vielleicht das Säurechlorid $\mathrm{ClCO}-\mathrm{C} \equiv \mathrm{G}-\mathrm{COCl}$ gewinnen liesse, welches präparativ wertvoll wäre; doch zeigte es sich, dass hierbei die Addition von Chlorwasserstoff unvermeidlich ist; es wurde das Säurechlorid der Chlorfumarsäure $\mathrm{ClCO}-\mathrm{CGl}=\mathrm{CH}-\mathrm{COCl}$ erhalten. - Thionylchlorid wirkt auch beim Kochen auf Acetylen-dicarbonsäure nicht nennenswert ein. Ein Versuch, aus Acetylen-dimagnesiumbromid und überschüssigem Phosgen nach der Gleichung

$$
\mathrm{ClCOCl}+\mathrm{BrMgC} \equiv \mathrm{C} \mathrm{MgBr}+\mathrm{ClCOCl} \rightarrow \mathrm{ClCO}-\mathrm{C} \equiv \mathrm{C}-\mathrm{COCl}+2 \mathrm{MgBrCl}
$$

das Säurechlorid zu erhalten, gab nur harzige Substanzen.

1) Lossen, A. 175, 313 (1874); vergl, auch Thiele und Pickard, A. 309, 189 (1899).

2) v. Rothenburg, I. pr. [2] 51, 53 (1895).

3) Vergl. Ruggli und Hartmann, Helv. 3, 493 (1920); Weerman, A. 401, $1(1913)$.

4) B. 18, $2271(1885)$. 
Es war nun naheliegend, die dreifache Bindung der Acetylendicarbonsäure provisorisch zu schützen durch Anlagerung von Atomen, welche sich später an geeigneter Stelle unter Regenerierung der Acetylenbindung wieder abspalten lassen. Hier kommen vorwiegend Halogen und Halogenwasserstoff in Betracht. Von den hieher gehörigen halogenierten Säuren ist die Dibrommaleïnsäure (Formel I) am leichtesten zugänglich und wurde in der vorangehenden Arbeit von P. Ruggli und C. Hartmann ${ }^{1}$ ) untersucht. Von den Abbauverfahren ist das über die Hydrazide und Azide bei ungesättigten Säuren das günstigste, doch führte es zu einem ringförmigen Hydrazid (Formel II), welches kein Azid mehr bilden kann.

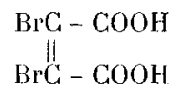

I.

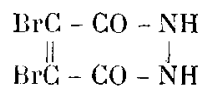

II.

Infolgedessen wurden die Versuche auf die Transform, die Dibromfumarsäure (Formel III) ausgedehnt. Hier ist leider die umständliche Beschaffung des Materials hinderlich, da diese Säure nur durch Addition von Brom an Acetylen-dicarbonsäure erhalten werden kann. Auch entstehen hierbei, wie $O t t^{2}$ ) nachwies, daneben meist einige Prozente Dibrommaleïnsäure, welche entfernt werden müssen. (Die Addition von Jod unter Bildung von Dijodfumarsäure ${ }^{3}$ ) verläuft nicht besonders glatt.) Bei Addition von Brom an Acetylen-dicarbonsäure st er entsteht ein schwer zu trennendes Gemisch von Dibromfumar- und Dibrommaleïnester ${ }^{4}$. Der naheliegende Gedanke, Dibrommaleïnsäure oder ihre Derivate in die Fumarform umzulagern, ist nicht ausführbar, da nach $\left.O t t^{5}\right)$ in diesem Ealle die Maleïnform die stabile ist. Immerhin findet sich in der Literatur eine Stelle ${ }^{6}$ ), wo angegeben wird, dass die Halogenmaleïnsäuren bei der Veresterung durch Spuren freien Jods in fumaroïde Formen übergehen, doch konnte ich bei einer Wiederholung dieser Versuche mit dem Ester auch unter absichtlichem Jodzusatz keinerlei Umlagerung; beobachten, sondern
1) Helv. 3, 493 (1920).
2) A. 392, $268(1912)$.
3) Bruck, B. 24, 4118 (1891); 26, 847 (1893).
4) Michael, J. pr. 46, 228 (1892).
5) A. 392, 250 (1912).
6) Salmony und Simonis, B. 38, 2581 (1905). 
nur die Ergebnisse von Ott in jeder Hinsicht bestätigen. Auch längere Belichtung konzentrierter wässriger Lösungen von Dibrommaleïnsäure oder ihrem Natriumsalz bewirkte keine nennenswerte Veränderung.

Es wurden dann die verschiedenen Möglichkeiten zur Darstellung von Acetylen-dicarbonsäure eingehend verglichen; dabei wurde das ursprüngliche Verfahren von Bandrousti $i^{1}$ ) und $v . B a e y e r^{2}$ ) (Fumarsäure $+\mathrm{Br}_{2},-2 \mathrm{HBr}$ ) als das beste befunden. Das theoretisch so hübsche Verfahren von Jocitch ${ }^{3}$ ), das in einer Umsetzung von Acetylen-dimagnesiumbromid $\mathrm{BrMgG} \equiv \mathrm{CMgBr}$ mit Kohlendioxyd besteht, gibt schlechte Ausbeuten und ein unreines Produkt. Ein Versuch, Natriumstaub unter Xylol mit Acetylen und Kohlendioxyd zu behandeln, gab keine nennenswerte Einwirkung. Es war nun denkbar, dass sich die leicht zugängliche Dibrommaleïnsäure (Formel I) durch Bromentziehung in Acetylen-dicarbonsäure verwandeln liesse; da jedoch bei Anwendung von Zinkstaub Wasser sowie saure Reaktion zu vermeiden ist, musste in Pyridinlösung resp. Aceton-pyridin gearbeitet werden, was wegen der Schwerlöslichkeit des entstehenden Pyridinsalzes im kalten Pyridin schon misslich ist und auch keine brauchbaren Resultate gab. Eher anwendbar ist die Entbromung des Dibommaleïnsäureesters mit Zinkstaub; sie führt zum Acetylen-dicarbonsäureester, doch erfordert die Reaktion so Ianges Kochen, dass ein Teil verharzt. Ich ziehe daher die direkte Veresterung der Acetylen-dicarbonsäure nach Michael vor.

Lässt man nun auf Dibromfumarsäureester Hydrazin wirken, so erhält man, ähnlich wie beim Dibrommaleïnsäureester ${ }^{4}$ ), tiefe Violettfärbung und Verharzung unter Eliminierung des Broms. Dagegen gelang es nach einer Reihe von Versuchen, aus dem Säurechlorid der Dibromfumarsäure $\mathrm{ClCO}-\mathrm{CBr}=\mathrm{CBr}-\mathrm{COCl}$ durch Umsetzung mit Hydrazinhydrat in Äther-Petroläther das schön krystallisierte Dihydrazid der Dibromfumarsäure (Formel IV) zu gewinnen.

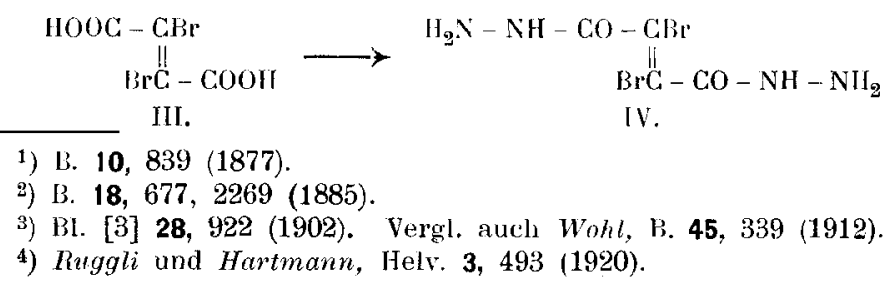

III.

IV.
1) B. 10,839 (1877).
2) B. 18,677, $2269(1885)$.
3) BI. [3] 28, 922 (1902). Vergl. auch Wohl, B. 45, 339 (1912).

4) Ruggli und Hartmann, Helv. 3, 493 (1920). 


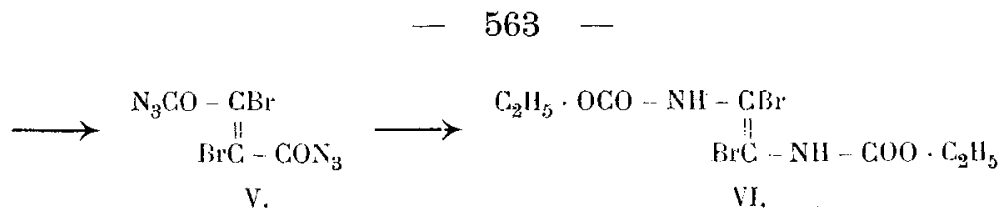

Leider konnte die Ausbeute nicht über 18 Prozent der Theorie gesteigert werden, da auch hier begreiflicherweise durch polymolekulare Reaktionen Bildung amorpher Produkte eintritt und auch das Hydrazid selber ziemlich empfindlich und leicht verseifbar ist. Die Anwendung besonderer Vorsichtsmassregeln, z. B. die Verwendung des in indifferenten Medien leichter löslichen wasserfreien Hydrazins, intensives Rühren oder Kühlen verbesserte die Ausbeute nicht. Einige Hoffnung setzte ich auf die schöne Methode von $G$. Schröter ${ }^{1}$ ), durch Umsatz der Säurechloride mit trocknem Natriumazid direkt zu den Säureaziden resp. unter Stickstoffabspaltung direkt $\mathrm{zu}$ den Isocyanaten ete. zu gelangen, doch wirkte Dibromfumarsäurechlorid auch in kochendem Ligroin resp. bis gegen $170^{\circ}$ nicht auf Natriumazid ein. Dies beruht offenbar auf dem starken Halogengehalt, wie ja auch Schröter beobachtete, dass das stark halogenhaltige Trichloracetylchlorid ausserordentlich schwer resp. unvollständig reagiert.

Dafür gelang die Reaktion leicht durch Umsetzung des Dibromfumarsäure-dihydrazids (IV) mit salpetriger Säure. Es wird als farbloser Niederschlag das ungeheuer explosive Diazid der Dibromfumarsäure (Formel V) erhalten. Verkocht man dieses mit Alkohol, so findet Stickstoffentwicklung statt und man erhält einen schön krystallisierenden Körper, den ich als den zweifachen Carbaminsäureester des Diamido-dibrom-äthylens der Formel VI anspreche. Leider war es mir, speziell infolge der geringen Ausbeuten an Dihydrazid, bisher nicht möglich, die zur Untersuchung erforderliche Menge dieses Körpers zu beschaffen. Doch zweifle ich nicht, dass, wenn das Material zu beschaffen ist, sich auch die Bromentziehung durchführen lässt und man zu einem Carbaminat des Diamido-acetylens $\mathrm{C}_{2} \mathrm{H}_{5} \mathrm{O} \cdot \mathrm{CO}-\mathrm{NH}-\mathrm{G} \equiv \mathrm{C}-\mathrm{NH}-\mathrm{COO} \cdot \mathrm{C}_{2} \mathrm{H}_{5}$ gelangt. -

Man kann noch weitere Reaktionsfolgen konstruieren, welche zu Diamido-acetylenderivaten führen können, und die zum Teil experimentell von mir beschritten wurden. So erschien es nicht aus-

1) B. 42,3357 (1909). 
geschlossen, die von Bamberger und Berlé $\left.e^{1}\right)$ beschriebene Aufspaltung des Imidazols mit Benzoylchlorid zu einem dibenzoylierten Diamido-äthylen

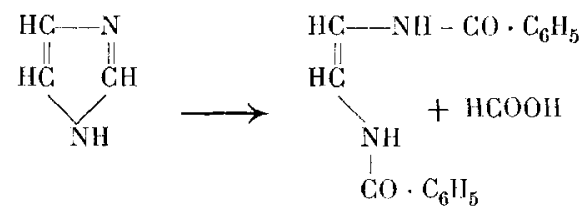

auf halogenierte Imidazole anzuwenden. Ich versuchte bisher, das Tribrom-imidazol zu einem Benzoylderivat des Dibromäthylens aufzuspalten,<smiles>CCCCCOc1ccccc1</smiles>

doch tritt beim Schütteln mit Benzoylchlorid und Natronlauge überhaupt keine Reaktion ein, während sich beim Arbeiten in Pyridinlösung ein normales Benzoylderivat bildet:

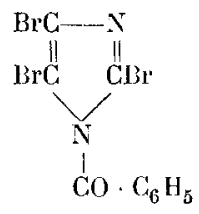

Die Versuche werden fortgesetzt.

\section{Experimentelles.}

Acetylen-dicarbonsäure.

$\mathrm{Zu}$ der Methode von Bandrowski $i^{2}$ ) und $v$. Baeyer ${ }^{3}$ ) seien noch folgende ergänzende Daten mitgeteilt, welche ein bequemes Arbeiten mit grösseren Mengen unter Erzielung eines reinen, ganz bromfreien Produkts gestatten: $100 \mathrm{gr}$ feingepulverte käufliche Fumarsäure werden in einer Sodawasserflasche mit $50 \mathrm{~cm}^{3}$ Brom in $100 \mathrm{~cm}^{3}$ Eisessig übergossen, mit dem Glasstab gut verrührt und verschlossen acht Stunden im kochenden Wasserbad

1) A. 273,342 (1893).

2) B. 10, 839 (1877); vergl. auch A. Michate, J, pr. [2] 52, 294 (1895).

3) B. 18, 677, 2269 (1885). 
erhitzt; der erkaltete Inhalt wird zerstossen, abgesaugt und mit insgesamt $150 \mathrm{~cm}^{3}$ Eisessig portionsweise verreibend gewaschen. Das scharf abgesaugte Produkt wird auf dem Wasserbad bis zur Farb- und Geruchlosigkeit getrocknet; Ausbeute 185-195 gr Dibrombernsteinsäure. Aus der Waschfluissigkeit lassen sich noch 7-10 gr gewinnen. Je $50 \mathrm{gr}$ hiervon werden mit etwas mehr als $4 \mathrm{Mol}$. konzentrierten methylalkoholischen Kalis fünf Viertelstunden am Rückflusskühler gekocht und nach Erkalten abgesaugt. Zwei solche Portionen werden gemeinsam mit $200 \mathrm{~cm}^{3}$ Alkohol verreibend gewaschen, abgesaugt und zwischen Filtrierpapier getrocknet; sie geben 130 bis $144 \mathrm{gr}$ Salzgemisch (= acetylendicarbonsaures Kalium + Kaliumbromid). Das Salzgemisch wird, je nach Ausbeute, in $260-280 \mathrm{~cm}^{3}$ Wasser gelöst und mit einer Mischung von $8-9 \mathrm{~cm}^{3}$ konzentrierter Schwefelsäure mit $30 \mathrm{~cm}^{3}$ Wasser (dreiviertel der berechneten Säuremenge) das saure Kaliumsalz der Acetylen-dicarbonsäure gefällt. Man lässt drei Stunden oder über Nacht stehen und saugt ab. (Das Filtrat gibt mit mehr Säure eine zweite Fällung, die aber zuweilen bromhaltig ist und daher nach Sammlung mehrerer Portionen in Alkali gelöst und mit äquivalenter Säuremenge als saures Kaliumsalz wieder ausgefällt wird.) Das saure acetylen-dicarbonsaure Kalium (1. Fällung) wird mit $40 \mathrm{~cm}^{3}$ Wasser gewaschen ${ }^{1}$ ), und ist dann praktisch bromfrei. Ausbeute $43-58 \mathrm{gr}$. Je $42 \mathrm{gr}$ werden mit einer Mischung von $60 \mathrm{~cm}^{3}$ konzentrierter Schwefelsäure und $240 \mathrm{~cm}^{3}$ Wasser versetzt und entweder acht Stunden im Apparat mit Äther extrahiert oder sechs bis sieben mal mit je $100 \mathrm{~cm}^{3}$ Äther ausgeschüttelt. Der Ätherrückstand wird einen Tag auf Ton oder Papier abgepresst und, falls er klebrig ist, mit wenigen $\mathrm{cm}^{3}$ kalten Äthers verreibend gewaschen. Ausbeute an krystallwasserhaltiger reiner Acetylendicarbonsäure 28-30 gr. Unter Mitbenutzung der zweiten Fällung des sauren Kaliumsalzes beträgt die Ausbeute aus 100 gr Fumarsäure etwa 70 gr Acetylendicarbonsäure. Im Vakuum uiber Schwefelsäure einen Tag getrocknet, zeigt sie, rasch erhitzt. den Zersetzungspunkt $177^{\circ}$.

$\mathrm{Zu}$ der Bildungsweise näch Jocitch ${ }^{2}$ ) sei erwähnt, dass als Verdünnungsmittel für das sirupöse Acetylen-dimagnesiumbromid Chloroform verwendet wurde. -

1) Das Waschwasser wird bei der Aufarbeitung der "zweiten Fällung" mit verwendet.

2) Bl. [3] 28, 922 (1902). 
Dibrommalë̈ns̈̈ure und Zinkstaub:

Als Lösungsmittel kam nux Pyridin in Betracht. Behandelt rnan die freie Säure (erhalten aus dem Anhydrid durch Verreiben mit kalter konzentrierter Salzsäure) mit Pyridin in der Wärme, so wird sie verharzt; in der Kälte entsteht das Pyridinsalz. Zu dessen Darstellung trägt man soviel Säure in gekühltes Pyridin ein, als sich darin auflöst, filtriert eventl. und lässt weiter in Eis stehen, wobei sich reichlich Krystalle des Monopyridinsalzes abscheiden, welche abgesaugt und luftrocken analysiert wurden. 0,0953 gr Subst verhrauchten, mit Alkali destilliert, $2,75 \mathrm{~cm}^{3} 0,1-$ n.-Säure $\mathrm{C}_{4} \mathrm{H}_{2} \mathrm{O}_{4} \mathrm{Br}_{2}, \mathrm{Py}$ Fier. Py $22,38 \%$ Gef. Py $22,79 \%$

Smp. $120^{\circ}$ (Zers.). Bei einer Serie von Wägungen des frisch abfiltrierten Salzes bis zur Gewichtskonstanz konnten keine Anzeichen für die Existenz eines neutralen Salzes gefunden werden. Da also auch in Pyridinlösung das saure Salz entsteht, ist es begreiflich, dass beim Behandeln mit Zinkstaub in Pyridinlösung die Substanz in den Zinkschlamm ging ${ }^{1}$ ) und nicht ohne Wasserstoffentwicklung aufgearbeitet werden konnte; es wurde etwas Fumarsäure erhalten.

Auch von der nicht-substituierten Maleïnsäure konnte Lutz $\left.z^{*}\right)$ nur das saure Pyridinsalz erhalten und zog deshalb eine einbasische Lactonformel für diese Säure in Betracht. Doch sind diese sauren Salze nach $P$. Pfeiffer $^{3}$ ), welcher zahlreiche ähnliche Beispiele untersuchte, durch innere koordinative Absättigung des einen Wasserstoffatoms an den Carbonylsauerstoff der andern Säuregruppe entstanden :

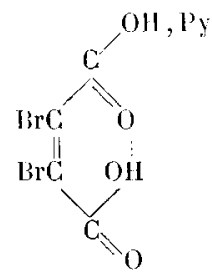

Es soll noch untersucht werden, ob sich das Salz in ein isomeres "Pyridinbetaïn" ${ }^{4}$ ) umlagern lässt.

1) Die überstehende Lösung enthielt last nichts.

2) B. 43, 2640 (1910); vergl. auch P. Pfeiffer und A. Langenberg, B. 43, $2926(1910)$.

3) B. 47,4580 (1914).

4) Vergl. Lutz, B. 43, 2636 (1910); Pfeiffer und Langenberg, B. 43, 2926 (1910). 
Acetylen-dicarbonsäure-diäthylester.

An einem nach Michä̈') durch Veresterung mit AlkoholSchwefelsäure gewonnenen Präparat wurde festgestellt, dass beim Kühlen mit Kältemischung der Ester krystallisiert; die Krystalle zeigen den Smp. $+1^{\circ}$ bis $2^{\circ}$. Die Dichte des flüssigen Esters wurde bestimmt $\mathrm{zu} \mathrm{d}_{4^{0}}^{15^{u}}=1,0690$.

Gewinnung des Acetylen-dicarbonsäureesters aus Dibrommaleïnsäureester: $13 \mathrm{gr}$ Dibrommaleïnsäureester in $30 \mathrm{~cm}^{3}$ Benzol wurden mit 6 gr 90prozentigem Zinkstaub unter Rückfluss gekocht. Nach einer Viertel- bis einer halben Stunde zeigt ein herausgenommener Tropfen beim Verdunsten den charakteristischen scharfen Geruch des Acetylen-dicarbonsäureesters. Nach sechzigstündigem Kochen wird filtriert und mit Äther nachgewaschen. Die Lösung gibt nach Abdestillieren der Lösungsmittel bei der Vakuumdestillation $2,1 \mathrm{gr}$ Acetylen-dicarbonsäureester $(30 \% \mathrm{der}$ Theorie), dann einen geringen Nachlauf, wahrscheinlich von unangegriffenem Dibrommaleïnsäureester; es bleiben 6 gr eines dunkeln, harzigen, nicht destillierbaren Rückstands. - Übrigens hat schon Michaël ${ }^{2}$ ) festgestellt, dass die Bromabspaltung beim Dibrommaleïnsäureester schwer erfolgt, 2,77 mal langsamer als beim Dibromfumarsäureester.

\section{Acetylen-dicarbonsäureester und Hydroxylamin.}

Die Reaktion verläuft heftig und erfordert daher Kühlung. Eine aus 6,62 gr Hydroxylaminchlorhydrat in $32 \mathrm{~cm}^{3}$ abs. Methylalkohol und einer Lösung von $2,19 \mathrm{gr}$ Natrium in $30 \mathrm{~cm}^{3}$ abs. Äthylalkohol bereitete Lösung von freiem Hydroxylamin (berechnet für beide Carboxylgruppen) wurde in Eis gekühlt und mit $8,1 \mathrm{gr}$ gleichfalls gekühltem Acetylen-dicarbonsäureester vorsichtig versetzt. Darauf wurde nochmals eine Lösung von 2,19 gr Natrium in $30 \mathrm{~cm}^{3}$ Alkohol hinzugegeben. Es schied sich ein gelbes Natriumsalz ab, welches am andern Tag abgesaugt und wiederholt mit abs. Alkohol, in dem es nicht unbeträchtlich löslich ist, gewaschen wurde; hierbei wurde es krystallinisch. Es wurde im Vakuum über Schwefelsäure in fünf Tagen gewichtskonstant; Ausbeute $7,8 \mathrm{gr}$. Es ist sehr hygroskopisch und löst sich in
1) J. pr. [2] 46, 224 (1892).
9) J. pr. [2] 52, 330 (1895). 
Wasser mit alkalischer Reaktion. Beim Erhitzen verpufft es harmlos. Die Substanz entfärbt Permanganat momentan. Mit Eisenchlorid gibt sie die für Hydroxamsäuren charakteristische intensive violette Färbung resp. Fällung. Versucht man durch Einwirkung verdünnter Säuren die freie Hydroxamsäure zu fassen, so erhält man nur Zersetzungsprodukte. Die Analyse erforderte in Anbetracht der leichten Verpuffung einige Vorsicht und gab bei verschiedenen Präparaten abweichende Werte. Von den Formeln VII und VIII ziehe ich trotz der grösseren Differenz die erstere wegen der typischen Hydroxamsäurereaktion vor; offenbar war dem Natriumsalz eine geringe Menge des entsprechenden Hydroxylaminsalzes und Feuchtigkeit beigemengt, worauf speziell die zu niedrigen Natriumwerte zurückzuführen sind.

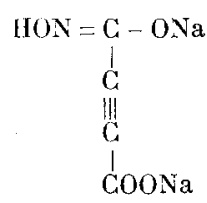

VII.

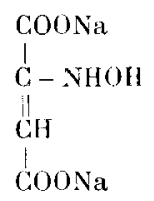

COONa

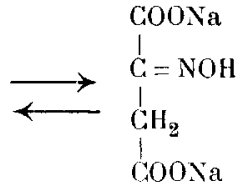

VIII.

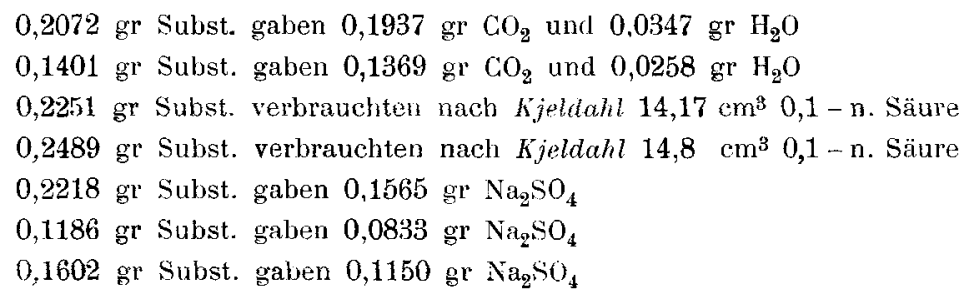

Formel VII, $\mathrm{C}_{4} \mathrm{IIO}_{4} \mathrm{NNa}_{2}$ Ber. C 27,74 II $0,58 \quad \times \quad 8,10 \quad \mathrm{Na} 26,59 \%$

Formel VIII, $\mathrm{C}_{4} \mathrm{H}_{3} \mathrm{O}_{5} \mathrm{NNa}_{2}$ Ber. ${ }^{2} 25,13$ n $1,58,7,33$, 24,08\%

Gef. "25,50 " $1,87,8,81$ " $22,85 \%$

"26.6.5 "2,06 " $8,36 \quad$ " $22,74 \%$

" $23,24 \%$

Mit angesäuerten Lösungen von Kupfersalzen entstehen olivgrüne, mit Silbernitrat gelbliche Fällungen.

Benzoylierungsversuche nach Schotten-Baumann gaben einmal geringe Mengen krystallisierender Körper, die beim Arbeiten mit grösseren Mengen nicht mehr erhalten werden konnten. 
 \\ Chlorfumarsäurechlorid.$$
\mathrm{CiCO}-\mathrm{CCl}=\mathrm{CH}-\mathrm{COCl}
$$

Dasselbe wurde bereits von $W . H$. Perkin $^{1}$ ) aus Weinsäure und Phosphorpentachlorid erhalten, aber nicht analysiert. Ich erhielt es aus Acetylen-dicarbonsäure auf folgende Weise: $4 \mathrm{gr}$ der wasserfreien Säure wurden mit 15 gr Phosphorpentachlorid unter Kühlung mit einer Kältemischung vermischt. Nach zwei Minuten trat lebhafte Reaktion unter Verfluissigung ein. Bei der Destillation im Vakuum gingen zuerst neben Phosphoroxychlorid bräunliche, leicht flüchtige Zersetzungsprodukte über, dann eine Hauptfraktion vom Sdp. $20 \mathrm{~mm} 70-80^{\circ}$; rektifiziert $2,7 \mathrm{gr}$. Sdp. $20 \mathrm{~mm} 73-75^{\circ}$.

0,2086 gr Subst. gaben 0,1940 gr $\mathrm{CO}_{2}$ und 0,0119 gr $\mathrm{H}_{2} \mathrm{O}$

$0,2174 \mathrm{gr}$ Subst. gaben $0,4960 \mathrm{gr} \mathrm{AgCl}$

$$
\begin{array}{lllll}
\mathrm{C}_{4} \mathrm{HO}_{2} \mathrm{Cl}_{3} & \text { Ber. C. } 25,62 & \text { H } & 0,54 & \text { Cl } 56,77 \% \\
& \text { Gef. \# } 25,36 & \text { " } & 0,64 & \text { " } 56,44 \%
\end{array}
$$

\section{Dibromfumarsäure}

wurde aus Acetylen-dicarbonsäure und Brom in der von E.Ott ${ }^{2}$ ) angegebenen Ausführungsform gewonnen und die etwa entstandenen Spuren von Dibrommaleïnsäure durch Vakuumsublimation entfernt. Man kann übrigens auch die Ausführungsform von Ott mit der von Lossen $^{3}$ ) kombinieren, indem man die Lösung der Acetylen-dicarbonsäure auf den Boden eines kleinen Exsikkators bringt und eine Schale mit der berechneten Menge Brom auf einem Glasdreifuss hineinstellt. Das ganze wird in einem grossen Topf mit Eis verpackt und an einem dunklen Ort stehen gelassen.

$$
\begin{gathered}
\text { Dibromfumarsäuredihydrazid. } \\
\mathrm{NH}_{2} \cdot \mathrm{NH}-\mathrm{CO}-\mathrm{CBr}=\mathrm{CBr}-\mathrm{CO}-\mathrm{NH} \cdot \mathrm{NH}_{2}
\end{gathered}
$$

2,1 gr Hydrazinhydrat werden in $30 \mathrm{~cm}^{3}$ absoluten Äther eingetragen und eine Lösung von 2,8 gr Dibromfumarsäurechlorid ${ }^{4}$ ) in $20 \mathrm{~cm}^{3}$ Petroläther unter flottem Rühren ziemlich rasch zuge493 (1920).

1) Soc. 53, 696 (1888); vergl. auch Ruggli und Hartmann, Helv. 3,

2) A. 392, 265 (1912).

4) E. Ott, A. 392, $268(1912)$.

3) A. $348,324(1906)$. 
geben. Man lässt die ziemlich lebhafte Reaktion unter beständigem Rühren oder Umschwenken vor sich gehen. Das herumschwimmende Öl erstarrt rasch zu einer bräunlichen halbfesten Masse und wird nach kurzem Stehen abgesaugt und auf Ton gestrichen, worauf man die Tonplatten eine halbe bis dreiviertel Stunden im Schwefelsäurexsikkator stehen lässt. Die eventl. nochmals gepulverte Masse wird dreimal mit je $50 \mathrm{~cm}^{3}$ wasserfreiem Methylalkohol ausgekocht und die filtrierten Lösungen, jede für sich, auf dem Wasserbad unter Zusatz von Tierkohle in der Schale bis auf ein kleines Volum (ca. $3-6 \mathrm{~cm}^{3}$ ) eingekocht, filtriert und unter Impfen mit dem Glasstab lebhaft gerieben. Wichtig ist, dass die Lösung möglichst konzentriert und entfärbt wurde; sie muss hellgelb sein, da dunkle Lösungen nicht krystallisieren. Die Krystalle werden nach einer halben Stunde abgesaugt und nach Waschen mit Methylalkohol auf Ton getrocknet. Ausbeute 0,3 bis $0,5 \mathrm{gr}$ an reiner Substanz. Dieselbe wurde nur für Analysenzwecke aus Alkohol umkrystallisiert; für präparative Zwecke ist dies nicht empfehlenswert, da grosse Verluste hierbei eintreten. Die Darstellung muss ohne Unterbrechung ausgefübrt werden.

$$
\begin{aligned}
& 0,1004 \text { gr Subst. gaben } 15,4 \mathrm{~cm}^{3} \mathrm{~N}_{2}\left(1^{0}, 759 \mathrm{~mm}\right) \\
& 0,0505 \mathrm{gr} \text { Subst. gaben } 0,0630 \mathrm{gr} \mathrm{AgRr} \\
& \mathrm{C}_{4} \mathrm{H}_{6} \mathrm{O}_{2} \mathrm{~N}_{4} \mathrm{Br} \mathbf{r}_{2} \quad \text { Ber. N 18,56 Br 52,93\% } \\
& \text { Gef. " } 18,41, \quad 53,09 \%
\end{aligned}
$$

Die Substanz zersetzt sich bei ca. $154^{0}$ unter Aufblähen. Schwerlöslich in Benzol, leichtlöslich in heissem Alkohol, mässig löslich in kaltem Wasser.

Bei der Darstellung des Dihydrazids geht der grösste Teil des Materials in ein braunes Pulver über, welches sehr schwer löslich ist und erst bei hoher Temperatur, ohne zu schmelzen, verkohlt.

$$
\begin{gathered}
\text { Dibromfumarsäure-diazid. } \\
\mathrm{N}_{3} \mathrm{CO}-\mathrm{CBBr}=\mathrm{CBr}-\mathrm{CON}_{3}
\end{gathered}
$$

Man löst das Dibromfumarsäure-dihydrazid in möglichst wenig kaltem Wasser und versetzt unter Eiskühlung mit etwas mehr als der berechneten Menge Natriumnitritlösung und ver- 
dünnter Essigsäure. Das Azid scheidet sich langsam als weisses Pulver $a b$ und wird nach dreiviertel- bis einstündigem Stehen in der Kälte abgesaugt und mit Wasser gewaschen. Auf Ton getrocknet, explodiert es bei leichtem Reiben mit grosser Heftigkeit; auf eine Analyse wurde daher verzichtet.

Beim Erwärmen mit Alkohol entsteht unter Stickstoffentwicklung eine krystallisierte Substanz, offenbar das Carbaminat des Diamido-dibrom-äthylens, welches noch zu untersuchen ist.

\section{Benzoyl-tribrom-imidazol}

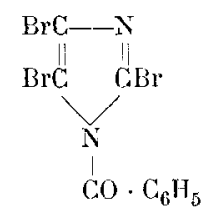

1 gr Tribrom-imidazol ${ }^{1}$ ) wurde in $7 \mathrm{~cm}^{3}$ Pyridin gelöst, mit Eiswasser gekühlt und mit $1,5 \mathrm{~cm}^{3}$ Benzoylchlorid tropfenweise versetzt. Nach mehrstündigem Stehen wurde die Masse in eisgekühlte verdünnte Schwefelsäure gegossen, wobei sich ein rotes Öl absetzte. Zur Entfernung von Benzoësäureanhydrid wurde die ganze Masse über Nacht auf der Maschine geschiuttelt und das jetzt noch vorhandene Öl mit Alkohol verrieben. Es krystallisierten 0,6 gr Benzoylderivat, welche aus Petroläther umkrystallisiert dicke Kryställchen vom Smp. $101-102^{\circ}$ ergaben.

$$
\begin{aligned}
& 0,2453 \text { gr Subst. gaben } 14, \tilde{5} \mathrm{crr}^{3} \mathrm{~N}_{2}\left(15,5^{\circ}, 753 \mathrm{~mm}\right) \\
& \qquad \mathrm{C}_{10} \mathrm{H}_{5} \mathrm{ON}_{2} \mathrm{Br}_{3} \text { Ber. N } 6,85 \% \\
& \\
& \text { Gef. }{ }^{\circ} 6,84 \%
\end{aligned}
$$

\section{Zusammenfassung:}

Auf der Suche nach Derivaten des Diamido-acetylens wurden die Acetylen-dicarbonsäure, die Dibrommaleïnsäure und die Dibromfumarsäure den Abbaumethoden unterworfen, welche in der Regel zum nächstniedrigeren Amin führen, und die hierbei erhaltenen

1) $W y s s, B .10,1370$ (1877). Das Tribrom-imidazol ist übrigens, entgegen der Literaturangabe, in Aether recht leicht löslich. 
Verbindungen beschrieben. Ein Ergebnis gibt nur die Hydrazidmethode; während Hydrazin mit Dibrommaleïnsäure ein ringförmiges Hydrazid gibt, konnte bei der Dibromfumarsäure ein Abbau, wenn auch noch unvollständig, erreicht werden.

Tribrom-imidazol wird durch Benzoylchlorid nicht, wie das Imidazol selber, zu einem Äthylenderivat aufgespalten, sondern benzoyliert.

$$
\begin{gathered}
\text { Basel, Anstalt für Organische Chemie, } \\
\text { im Mai } 1920 .
\end{gathered}
$$

\section{Errata.}

Helv. 3, 255, Zeile 11 v. o. statt , $\beta$-Tetracetyl-glucosidosalicylsäuremethylester" lies , $\beta$-Glucosido-salicylsäuremethylester.

Helv. 3, 255, Zeile 22 und 23 v. o. die angeführte Polarisation ist $\mathrm{zu}$ streichen. 